\title{
BMJ Global Health Conceptualising Long COVID as an episodic health condition
}

To cite: Brown DA, O'Brien KK. Conceptualising Long COVID as an episodic health condition. BMJ Global Health 2021;6:e007004. doi:10.1136/ bmjgh-2021-007004

Handling editor Seye Abimbola

Received 26 July 2021 Accepted 31 August 2021

Check for updates

(c) Author(s) (or their employer(s)) 2021. Re-use permitted under CC BY-NC. No commercial re-use. See rights and permissions. Published by BMJ.

${ }^{1}$ Therapies Department, Chelsea and Westminster Hospital NHS Foundation Trust, London, UK ${ }^{2}$ Department of Physical Therapy, Temerty Faculty of Medicine, University of Toronto, Toronto, Ontario, Canada ${ }^{3}$ Institute of Health Policy, Management and Evaluation (IHPME), University of Toronto, Toronto, Ontario, Canada ${ }^{4}$ Rehabiltation Sciences Institute (RSI), University of Toronto, Toronto, Ontario, Canada

Correspondence to Dr Kelly K O'Brien; kelly.obrien@utoronto.ca

\section{INTRODUCTION}

Globally growing numbers of individuals are living with persistent signs and symptoms following infection consistent with COVID19, referred to as Long COVID or PostCOVID Conditions. An estimated $6.2 \%$ of the $\mathrm{U} \mathrm{K}$ population self-identified experiencing Long COVID, which negatively affected their general well-being and ability to exercise and work. ${ }^{1}$ Long COVID symptoms also adversely affected day-to-day activities among $64.7 \%$ of those self-reporting Long COVID, with $19.3 \%$ reporting significant limitations to daily activities. $^{2}$

The long-term trajectory of Long COVID remains unknown. Therefore, conceptualising disability in Long COVID is essential for better understanding the lived experiences and health-related challenges of people living with and affected by Long COVID, to inform effective rehabilitation approaches and interventions to enhance clinical practice, policy and research. Our aim is to conceptualise Long COVID as an episodic health condition resulting in disability that may be characterised as multidimensional, episodic and unpredictable in nature and to highlight future directions, including the role for rehabilitation.

\section{LONG COVID AND EPISODIC DISABILITY}

Long COVID is characterised as a 'fluctuating' or 'relapsing and remitting' condition, ${ }^{3}$ which may be termed episodic. ${ }^{4}$ Episodes may fluctuate with Long COVID within the day, between weeks or over a longer continuum. Individuals can present with clusters of overlapping and episodic symptoms such as fatigue or exhaustion, postexertional symptom exacerbation, chest pain, painful joints or muscles, new shortness of breath and difficulty thinking or concentrating ('brain fog'). Individuals living with Long COVID sometimes characterise their experiences as unpredictable in nature. ${ }^{46}$ The multidimensional,

\section{Summary box}

Long COVID presents with multidimensional clusters of symptoms that can fluctuate, sometimes unpredictably, whereby symptoms adversely affect general well-being and ability to perform day-to-day activities, exercise or work.

- We propose that Long COVID should be conceptualised as an episodic illness, characterised by health-related challenges (or disability) that may be multidimensional, episodic and unpredictable in nature.

- Dimensions of disability experienced by people living with Long COVID may include physical, cognitive mental and emotional health, daily activities, social inclusion and uncertainty, with uncertainty and worrying about the future a key dimension of disability.

- The role for rehabilitation is important for people living with Long COVID, offering a goal-oriented, person-centred approach to prevent, mitigate and address episodic disability.

- Future directions include establishing valid and reliable measures of episodic disability, assessing effectiveness of rehabilitation approaches and interventions and building on existing international collaborations and community-engaged partnerships to advance practice, research and policy.

episodic and unpredictable health consequences of Long COVID resembles episodic disability, a concept derived from the context of HIV, where health challenges can fluctuate daily or over longer periods of time. ${ }^{78}$ There is an opportunity to apply lessons learned in other chronic and episodic illnesses to understand and conceptualise the disability experienced among adults living with Long COVID.

The dimensions of Long COVID disability can be represented by a diversity of healthrelated challenges spanning physical health challenges (fatigue or exhaustion, postexertional symptom exacerbation or shortness of breath), cognitive health challenges (difficulty thinking or concentrating ("brain fog')), mental-emotional health challenges (post-traumatic stress, anxiety or depression), difficulties carrying out day-to-day activities 
(showering, meal preparation or walking) and challenges to social inclusion (meaningful life roles or employment). The multidimensional nature of disability experienced by people living with Long COVID can adversely affect day-to-day activities, return to employment, family life, relationships, and ability to care for others. ${ }^{12}{ }^{4}$ Diagnostic clarity of Long COVID can be difficult to ascertain, particularly for those without a COVID-19 PCR, antigen or antibody test. Stigma related to disbelief of symptoms and the complexity of diagnostic uncertainty, particularly among those without a COVID-19 PCR, antigen or antibody test, can add complexity to those faced with challenges accessing employment or income benefits after leaving the workforce during illness, further contributing to disability and reduced quality of life after acute COVID-19. ${ }^{9}$

Uncertainty and worrying about the future is an important feature of living with Long COVID. Uncertainty was found to be a key dimension of disability and predictor of mental-emotional health and social inclusion for adults living with HIV. ${ }^{10}{ }^{11}$ In Long COVID, the impact of living with the uncertainty of when an episode of disability might arise, the severity and duration of that episode and the long-term implications on health are unknown. Disability frameworks are foundational for conceptualisation and accurately measuring health concepts in practice. Using an existing framework of disability developed from others living with episodic illness can provide a foundation for understanding disability experienced among people living with Long COVID.

\section{Episodic Disability Framework}

The Episodic Disability Framework was derived from the perspectives of people living with HIV in Canada to characterise the multidimensional and sometimes fluctuating nature of health challenges. ${ }^{7}$ The Episodic Disability Framework includes: (1) dimensions of disability (physical, cognitive, mental and emotional health, daily activities, social inclusion and uncertainty); (2) contextual factors, which include extrinsic (eg, support, environment and stigma) and intrinsic (self-care living strategies and personal attributes) factors that influence disability and (3) triggers, which can exacerbate a momentous episode. ${ }^{7}$ Empirically validated with adults living with HIV, the Episodic Disability Framework provides a novel method for conceptualising the multidimensional nature of living with chronic illness by capturing experiences of uncertainty and the temporal (fluctuating) feature of disability over time. ${ }^{12}$

We argue that this Framework may be an ideal approach from which to conceptualise disability experienced by people living with Long COVID. For instance, someone living with Long COVID may experience dimensions of disability, such as fatigue or exhaustion (physical) and brain fog (cognitive), impacting the ability to shower, prepare meals (daily activities) or return to work (social inclusion), resulting in low mood or depression

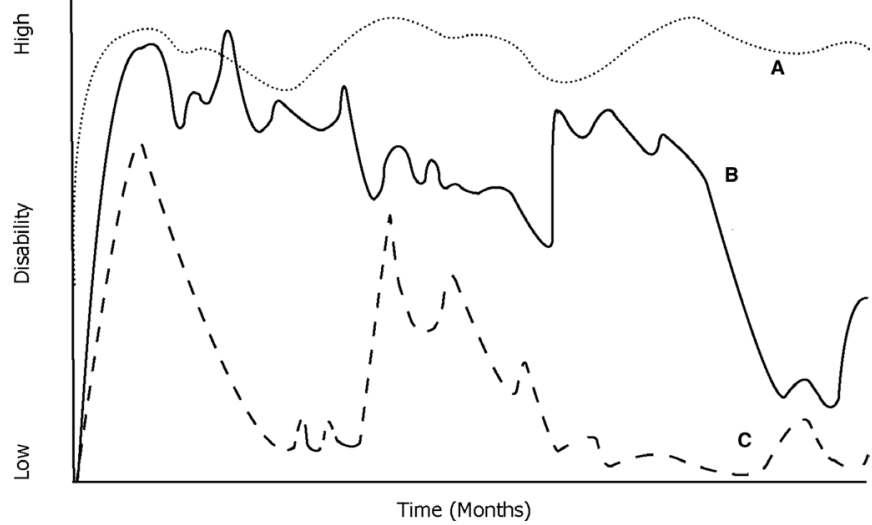

Figure 1 Examples of potential trajectories of episodic disability in people living with Long COVID. (A) Person with pre-existing disability whose disability is exacerbated by acute COVID-19, with disability remaining high during Long COVID with fluctuations over time. Presents with no trajectory of improvement over time. (B) Person experiencing new high level of disability during acute COVID-19, with continued episodic disability during Long COVID. Presents with a trajectory of episodes of limited improvement over time. (C) Person experiencing new moderate level of disability during acute COVID-19, who recovers after acute illness but during Long COVID experiences triggers that result in development of returning episodic disability. Presents with a slow trajectory of improvement over time.

(mental-emotional), all of which occur in combination with ongoing worry about how long these challenges will last and the long-term financial, practical and emotional consequences (uncertainty). These disability dimensions may be influenced by contextual factors, exacerbated (made worse) by stigma such as lack of belief of health challenges or alleviated (made better) by practical and emotional support (eg, peer support) and rehabilitation services. Furthermore, disability dimensions can be triggered by factors such as physical activity or exercise, prolonged cognitive activities (eg, online meetings) or other health conditions. Examples of potential trajectories of episodic disability that may be experienced among people living with Long COVID are illustrated in figure 1.

\section{FUTURE DIRECTIONS}

Opportunities exist to better understand and measure Long COVID disability, and to better direct and measure the effect of rehabilitation interventions in order to inform programmes and policies across global health populations. It will be critical to build on lessons learned in other chronic and episodic illnesses. Given the importance of terminology to provide clarity of understanding and communication among community and health providers, we recommend the use of the term 'episodic disability' to characterise the multidimensional, episodic and unpredictable health-related challenges associated with Long COVID.

We need to establish a better understanding of the lived experiences of people living with Long COVID, in 
order to provide a foundation for conceptualising and defining episodic disability experienced among people living with and affected by Long COVID. With clear conceptualisation and definition, the field will be better positioned to measure episodic disability to establish and evaluate the effectiveness of interventions in which to address episodic disability and enhance health outcomes.

\section{Measurement of episodic disability in Long COVID}

To provide optimal care in the context of Long COVID, we need to develop comprehensive, feasible tools to understand the profile and episodic nature of disability over time. Using a disability inclusive approach with standardised patient reported outcome measures (PROMs) designed to capture the nature and extent of disability and its fluctuation over time is critical to guide the provision of care, determine the effectiveness of interventions, and to inform workplace policies (eg, phased return to work), benefits, and access to services (eg, rehabilitation) for people living with Long COVID. ${ }^{9}$ New and existing measures of disability used in Long COVID, such as the COVID-19 Yorkshire Rehabilitation Scale, ${ }^{13}$ Long COVID symptom and impact tools ${ }^{14}$ and 12-item World Health Organization (WHO) Disability Assessment Schedule included within WHO case report form, ${ }^{15}$ do not describe the full breadth and depth of episodic disability. These measures do not capture the uncertainty dimension of disability (worrying about future health living with Long COVID) and do not consider the potential fluctuating nature of disability over time. Existing measures of disability established from other episodic illnesses that describe a range of health challenges a person might experience and the extent of fluctuation can provide a foundation for measuring episodic disability in the context of Long COVID. The HIV Disability (now Episodic Disability) Questionnaire was the first known measure of episodic disability, addressing gaps in previously existing health status measures to capture uncertainty and elements of social inclusion (relationships and employment) ${ }^{16}$ There is an opportunity to build from existing validated instruments in episodic disability with other chronic episodic conditions, such as HIV, to identify key features of disability specific to persons living with Long COVID, in order to help indicate areas in which to direct supports, interventions and resources. Given psychometric properties of PROMs are specific to the target population in which they were developed and assessed, evaluation of the Episodic Disability Questionnaire with people living with Long COVID is vital.

\section{Role for rehabilitation}

One key area in which to prevent, mitigate or address disability is rehabilitation. Rehabilitation is focused on the physical, mental and emotional, cognitive and social domains of health and disability and hence is well positioned to address episodic and multidimensional disability among people living with Long COVID. ${ }^{17}$ Rehabilitation involves any services or providers who address or prevent disability experienced by people living with chronic episodic illness. Rehabilitation should be disability focused, goal oriented, person centred, focused on function and tailored to an individual's goals, abilities and interests. ${ }^{4}$ There is a critical need to assess the impact of rehabilitation interventions to reduce episodic disability and enhance health outcomes for people living with Long COVID. Development of a robust research agenda involving disability and rehabilitation will be important for moving forward.

\section{International collaborations and community partnerships}

International partnerships and collaborations fostered in other health conditions provide a strong foundation from which to establish a coordinated, relevant and meaningful approach for advancing rehabilitation for people living with Long COVID. ${ }^{18}$ Access to timely, safe, effective and accessible rehabilitation is a critical area in need of development and highlights the opportunities for a 'strength in numbers approach' with other chronic and episodic illnesses to advance policy and programming related to Long COVID rehabilitation.

\section{CONCLUSION}

Long COVID may be conceptualised as an episodic illness, characterised by health-related challenges (or disability) that may be multidimensional, episodic and unpredictable in nature. It is essential to better understand and define episodic disability experienced by people living with Long COVID and establish tools to measure the presence, severity and episodic nature of disability, in order to target timely and appropriate rehabilitation interventions and mitigate disability to enhance health outcomes for people living with Long COVID. The role for safe and effective rehabilitation is emerging in the context of Long COVID. ${ }^{4}$ While evidence continues to develop, rehabilitation professionals are well positioned to address episodic disability in Long COVID. Opportunities exist to build on successful disability and rehabilitation models from other chronic and episodic illnesses in the context of Long COVID.

Twitter Darren A Brown @darrenabrown and Kelly K 0’Brien @kellyobrien25

Contributors Both authors contributed to the conceptualisation, writing and manuscript review.

Funding KKO is supported by a Canada Research Chair in Episodic Disability and Rehabilitation from the Canada Research Chairs Program.

Competing interests None declared.

Patient consent for publication Not required.

Provenance and peer review Not commissioned; externally peer reviewed.

Data availability statement There are no data in this work.

Open access This is an open access article distributed in accordance with the Creative Commons Attribution Non Commercial (CC BY-NC 4.0) license, which permits others to distribute, remix, adapt, build upon this work non-commercially, and license their derivative works on different terms, provided the original work is properly cited, appropriate credit is given, any changes made indicated, and the use is non-commercial. See: http://creativecommons.org/licenses/by-nc/4.0/.

\section{ORCID iDs}

Darren A Brown http://orcid.org/0000-0002-4956-243X 
Kelly K O’Brien http://orcid.org/0000-0002-1632-6537

\section{REFERENCES}

1 Office for National Statistics. Coronavirus and the social impacts of 'Long COVID' on people's lives in Great Britain: 7 April to 13 June 2021.

2 Office for National Statistics. Prevalence of ongoing symptoms following coronavirus (COVID-19) infection in the UK: 5 August 2021.

3 Ziauddeen N, Gurdasani D, O'Hara ME. Characteristics of Long COVID: findings from a social media survey. medRxiv 2021.

4 World Physiotherapy Response to COVID-19 Briefing Paper 9. Safe rehabilitation approaches for people living with Long COVID: physical activity and exercise. London, UK: World Physiotherapy, 2021. ISBN: 978-1-914952-00-5.

5 Centres for Disease Control and Prevention. Post-COVID conditions: information for healthcare providers: July 2021.

6 Davis HE, Assaf GS, McCorkell L, et al. Characterizing Long COVID in an international cohort: 7 months of symptoms and their impact. EClinicalMedicine 2021;38:101019.

7 O'Brien KK, Bayoumi AM, Strike C, et al. Exploring disability from the perspective of adults living with HIV/AIDS: development of a conceptual framework. Health Qual Life Outcomes 2008;6:76.

8 Solomon P, O'Brien KK, Nixon S, et al. Trajectories of episodic disability in people aging with HIV: a longitudinal qualitative study. $J$ Int Assoc Provid AIDS Care 2018;17:232595821875921.

9 Rayner C, Lokugamage A, Molokhia M. COVID-19: prolonged and relapsing course of illness has implications for returning workers. The BMJ Opinion 2020.
10 O'Brien KK, Hanna S, Solomon P, et al. Characterizing the disability experience among adults living with HIV: a structural equation model using the HIV disability questionnaire (HDQ) within the HIV, health and rehabilitation survey. BMC Infect Dis 2019;19:594.

11 Solomon P, O'Brien K, Wilkins S, et al. Aging with HIV and disability: the role of uncertainty. AIDS Care 2014;26:240-5.

12 O'Brien KK, Hanna S, Gardner S, et al. Validation of the episodic disability framework with adults living with HIV. Disabil Rehabil 2014;36:319-29.

13 Preston NJ, Parkin A, Makower S. The COVID-19 Yorkshire rehabilitation scale (C19-YRS): application and psychometric analysis in a post-COVID-19 syndrome cohort. medRxiv 2021.

14 Tran V-T, Riveros C, Clepier B, et al. Development and validation of the Long COVID symptom and impact tools, a set of patientreported instruments constructed from patients' lived experience. Clin Infect Dis 2021:ciab352.

15 World Health Organization. Global COVID-19 clinical platform case report form (CRF) for post COVID condition (post COVID-19 CRF) 2021.

16 O'Brien KK, Solomon P, Bergin C, et al. Reliability and validity of a new HIV-specific questionnaire with adults living with HIV in Canada and ireland: the HIV disability questionnaire (HDQ). Health Qual Life Outcomes 2015;13:124.

17 National Institute for Health Research. Living with COVID19 Second Review 2021.

18 Brown DA, O'Brien KK, Josh J, et al. Six lessons for COVID-19 rehabilitation from HIV rehabilitation. Phys Ther 2020;100:1906-9. 